\title{
Construcción de la feminidad en adolescentes de La Dorada, Caldas*
}

Construction of the femininity in adolescents from 12 to 17 years, La Dorada municipality of Caldas

Construção da feminidade em adolescentes de 12 a 17 anos da Dorada, Caldas

Recibido el 17 de enero de 2014, aceptado el 3 de abril de 2014

\author{
Diana Patricia Jaramillo Ortegón** \\ Colombia \\ Milena Giraldo Patiño*** \\ Colombia \\ Dora Cardona Rivas**** \\ Colombia
}

\section{Resumen}

, Para citar este artículo:

Jaramillo Ortegón, Diana,

Giraldo Patiño, Milena y

Cardona Rivas, Dora (2014).

Construcción de la feminidad en adolescentes de La Dorada,

Caldas. Ánfora, 21(36), 165-185.

Universidad Autónoma de Manizales. ISSN 0121-6538.
Objetivo: comprender el proceso de construcción de la feminidad en adolescentes del municipio La Dorada, Caldas. Metodología: estudio cualitativo que se aplicó en un grupo de adolescentes entre 12 y 17 años de los estratos 1 y 2 , del Colegio Renán Barco de los grados $7^{\circ}$ a $11^{\circ}$ de secundaria, y con la "Fundación Apoyar" de La Dorada, Caldas. A través de entrevista y grupos focales se indagó sobre experiencias, conocimientos e influencias que viven las adolescentes en el proceso de construir su feminidad. Resultados: se

\footnotetext{
* Trabajo resultado de tesis de Maestría en Salud Pública. La investigación fue denominada "Construcción de la Feminidad en adolescentes de 12 a 17 años de La Dorada (Caldas) realizada entre 2011 y 2012. Sustentada en 2013. Universidad Autónoma de Manizales.

** Fisioterapeuta. Departamento Movimiento Humano. Universidad Autónoma de Manizales, Colombia. djaramillo@autonoma.edu.co

** Bacterióloga. Universidad Católica de Manizales, Colombia. Milenita20@hotmail.com

**Ph D en Ciencias Sociales. Universidad Autónoma de Manizales, Colombia. dcrivas@autonoma.edu.co
} 
identificaron dos tipos de mediaciones que muestran cómo se construye la feminidad en las adolescentes de La Dorada: una mediación cultural y una mediación personal. Se evidenció, además, que las características atribuibles a la feminidad están dadas por los rasgos y las capacidades físicas de procreación, determinadas por su sexo y por las características de personalidad y comportamiento. Conclusiones: la construcción de la feminidad se encuentra "cautiva aún" de las representaciones que mantienen la dependencia de la mujer. Las estrategias formuladas en los programas de Salud Sexual y Reproductiva reclaman perspectivas que tengan en cuenta la realidad subjetiva de las adolescentes y su participación en la formulación de las políticas que les competen.

Palabras claves: Feminidad, Adolescentes, Identidad de género.

\title{
Resumo
}

Objetivo: compreender o processo de construção da feminidade em adolescentes de 12 a 17 anos, do Município da Dorada, Caldas. Metodologia: estudo qualitativo que se aplicou em um grupo de adolescentes entre 12 e 17 anos dos estratos 1 e 2, do Colégio Renan Barco da serie $7^{\circ}$ até $11^{\circ}$ do colégio, e com a "Fundação Apoiar" da Dorada, Caldas. O través de entrevistas e grupos focais se indagou sobre experiências, conhecimentos e influencias que vivem as adolescentes no processo de construção da sua feminidade. Resultados: identificaram se dois tipos de mediações que amostram como se constrói a feminidade nas adolescentes da Dorada: uma mediação cultural e uma mediação pessoal. Evidenciaram se, além que as características atribuíveis à feminidade estão dadas pelos riscos e as capacidades físicas de procriação, determinadas pelo seu sexo e pelas características de personalidade e comportamento. Conclusões: a construção da feminidade se acha "cativa ainda" das representações que matem a dependência da mulher. As estratégias formuladas nos programas de Saude Sexual e Reprodutivas reclamam perspectivas que tenham em conta a realidade subjetiva das adolescentes e sua participação na formulação das políticas que Ihes competem.

Palavras chaves: Feminidade, Adolescentes, Identidade de gênero.

\begin{abstract}
Objectives: to determine the relation between learning styles and academic performance in 2012 second term Nursing students of Universidad Cooperativa de Colombia, with its headquarters in Santa Marta. Methodology: a 186-students sample was taken. They were administered the Honey- Alonso learning style questionnaire. In addition, grade records were used to obtain their cumulative averages. Results: it was found that there are no statistically significant differences amongst the means of students' academic performance. Reflexive was the preferred learning style, with a 15,61 mean. The standard deviation of the four styles was between 2,56 and 3,3. The percentage
\end{abstract}


distribution of learning styles preferred by students, which were included in this study, was: active $21 \%$, reflexive $13,5 \%$, theoretical $39,5 \%$ and pragmatic $26 \%$. Conclusions: there was no significant influence of learning styles in academic performance of students who participated in this study. This study also concludes that some factors, such as personal and familial characteristics, as well as teachers' teaching style, affect students' academic performance.

Key words: Learning styles, Academic performance, Apprenticeship. 


\section{Introducción}

La salud sexual y reproductiva de la mujer hace parte de las preocupaciones todos los pueblos del mundo. Desde 1994, con la Conferencia Internacional sobre Población y Desarrollo (CIPD), realizada en El Cairo con el auspicio de las Naciones Unidas, se trazaron objetivos para potenciar a la mujer y propiciar la satisfacción de sus necesidades de educación y salud, incluida la salud reproductiva. Para ello, se adoptó un programa para avanzar en la igualdad de género, eliminar la violencia contra la mujer y garantizar la capacidad de la mujer para controlar su propia fecundidad.

En Salud Sexual y Reproductiva (SSR), se ha propiciado la planificación familiar, la asistencia del parto y la prevención de infecciones de transmisión sexual y el VIH/SIDA. Igualmente, hubo pronunciamientos en la IV Conferencia Mundial sobre la Mujer, celebrada en Beijing en 1995 y la Cumbre del Milenio de 2000. Para el cumplimiento de los objetivos de Salud, el Fondo de Poblaciones de Naciones Unidas (UNFPA) acompañó a Colombia en el diseño y la implementación de políticas en salud sexual y reproductiva, apoyó la creación y el fortalecimiento de capacidades institucionales para apropiar las metas de salud sexual y reproductiva en los planes territoriales de salud de los municipios y los departamentos, con base en la Política Nacional de Salud Pública 2007-2010.

Uno de los fenómenos más preocupante es el alto nivel de embarazos de adolescentes. De acuerdo con los resultados de las Encuestas de Demografía y Salud en Colombia, la proporción de adolescentes madres o embarazadas se incrementó significativamente entre 1990 y 2010 que pasó de $12,8 \%$ a 19,5 \%, aunque en los últimos cinco años presenta una leve tendencia a la baja. La propensión al aumento del embarazo adolescente en las últimas décadas, se observa en áreas urbanas y rurales y en mujeres de diferente nivel socioeconómico (Flórez y Soto, 2013).

Investigaciones, como las de Flórez y Soto (2007), han señalado que la fecundidad adolescente, del mismo modo que la fecundidad global, está determinada por factores individuales, interpersonales y contextuales que actúan a través de los determinantes próximos: inicio de relaciones sexuales, uso de métodos de control natal y aborto, principalmente. Señalan, igualmente, que el proceso ha estado acompañado por un descenso significativo en la edad de inicio de las relaciones sexuales.

En el diseño y la implementación de alternativas de solución a este problema, el Fondo de Población de las Naciones Unidas (UNFPA) acompaña a Colombia en el montaje de servicios en salud amigables para adolescentes y jóvenes, de acuerdo con el modelo diseñado por el Fondo, en conjunto con el Ministerio de la Protección Social, que fue impulsado desde 2008. 
Jaramillo Ortegón, Diana, Giraldo Patiño, Milena y Cardona Rivas, Dora (2014). Construcción de la feminidad en adolescentes de La Dorada, Caldas. Ánfora, 21(36), 165-185. Universidad Autónoma de Manizales. ISSN 0121-6538.

Algunas normas en salud sexual y reproductiva han sido:

> Inclusión de los métodos anticonceptivos hormonales y el condón masculino en el plan de beneficios del Plan Obligatorio de Salud -POS- del régimen contributivo y subsidiado (Acuerdo 380 de 2006).

> Autorización de la interrupción voluntaria del embarazo, en los casos en que la concepción haya sido producto de una violación, cuando existan malformaciones en el feto incompatibles con la vida o cuando el embarazo ponga en peligro la vida de la madre (Sentencia C-355 de 2006).

Adicionalmente, con la expedición de la Ley de Infancia y Adolescencia (Ley 1098 de 2006) se genera un escenario propicio para abogar por los derechos sexuales y reproductivos de los jóvenes. Y el Plan Nacional de Desarrollo 2006-2010 incluye, explícitamente, la igualdad de género entre sus objetivos.

Además, para resolver los problemas relacionados con la Salud Sexual y Reproductiva(SSR) se han establecido políticas que pretenden mejorar la salud sexual y reproductiva y promover el ejercicio de los derechos sexuales y reproductivos de la población con especial énfasis en la reducción de los factores de vulnerabilidad y los comportamientos de riesgo, el estímulo de los factores protectores y la atención a grupos con necesidades específicas de las mujeres.

Las metas apuntan a la reducción del embarazo adolescente, la reducción de la tasa de mortalidad materna evitable, el cubrimiento de la demanda en planificación familiar, la detección temprana del cáncer de cuello uterino, la prevención y atención de las ITS, el VIH y el SIDA y la detección y atención de la violencia doméstica y sexual. La política de Salud Sexual y Reproductiva (SSR) también incluye un aparte sobre las posibles fuentes de financiación para su aplicación, un mapa de competencias y responsabilidades que incluye a los principales actores del sector y de otros sectores respecto a la SSR.

Las estrategias planteadas por la Política son: promoción de la salud mediante acciones de información, educación y comunicación, la coordinación intersectorial e interinstitucional, el fortalecimiento de la gestión institucional, el fortalecimiento de la participación social, el desarrollo de la investigación y la potenciación de las redes sociales de apoyo. Para estas estrategias se han presentado respectivas líneas de acción que las desarrollan.

Ahora, se ha señalado que la reforma al Sistema de Salud Colombiano ha contribuido al desarrollo de la Política de Salud Sexual y Reproductiva, al favorecer el acceso de la población a los servicios de SSR, lo que ha llegado a ser una prioridad del Plan Nacional de Salud Pública (PNSP).

En el Eje Cafetero, la investigación sobre la implementación dicha política mostró una reducción en el número de nacimientos de adolescentes (10-19 años) entre 2003 y 2005. Sin embargo, no se alcanzaron a determinar las intervenciones específicas que permitieron obtener esta reducción. Los investigadores lo atribuyen a las diferentes metodologías empleadas. Sin embargo, parte de los logros reportados 
en los tres departamentos consiste en haber visibilizado a los adolescentes como una población prioritaria para el Eje Cafetero, más allá de la situación problemática de los embarazos y el VIH-SIDA (Castillo, et al., 2008).

Aunque la estrategia central es la Promoción de la Salud, los componentes de la política y los programas se orientan por una visión biologista y medicalizada de la sexualidad y no como un campo en el que se articulan componentes sociales, culturales, políticos y económicos. Esta visión se expresa en el énfasis para el control de la fecundidad adolescente a través del uso de anticonceptivos o el aborto en condiciones especiales y en el que la estrategia información-educación-comunicación no tiene efectividad comprobada.

Desde los 90, se ha cuestionado dicha visión y sólo recientemente se ha reconocido la importancia de los patrones de significado que contribuyen a la configuración del hacerse hombre, hacerse mujer y el aporte del conocimiento de tales significados a la comprensión de las preocupaciones de la sociedad sobre la vida sexual de los adolescentes.

A tales significados no es posible acceder desde perspectivas epistemológicasmetodológicas objetivistas, pues, más bien requiere abordajes que incluyan las relaciones sociales, económicas y políticas, como mediadoras en la construcción de los sujetos individuales y colectivos.

Los análisis realizados sobre el comportamiento sexual, los conocimientos y las prácticas sexuales, han proporcionado elementos de orden cuantitativo que indican que los adolescentes, al menos en el área urbana, cuentan con los conocimientos sobre su sexualidad, los medios para tener relaciones sexuales sin riesgo, sin que, al parecer, tales conocimientos contribuyan al cambio comportamental requerido para proteger su salud y controlar reflexivamente la reproductividad.

Por eso, los acercamientos socioculturales al estudio de la población adolescente indican que la socialización primaria y secundaria y las condiciones estructurales de la sociedad influyen en la construcción de los significados de la sexualidad en las personas (Steve, 2005) y que son estos significados subjetivos los que orientan su vida sexual y reproductiva. Esta última perspectiva origina la presente investigación, en la cual se pretende comprender cómo ocurre el proceso de construcción de la feminidad en adolescentes de 12 a 17 años del municipio de la Dorada, a partir de los significados, los conocimientos, las experiencias y las influencias vividas por este grupo en el proceso de convertirse en mujeres.

\section{Metodología}

Se diseñó un estudio con enfoque cualitativo, a través del cual se indagó por los conocimientos, prácticas y experiencias vividas por las adolescentes durante el desarrollo de su feminidad con el propósito de develar los significados simbólicos observados en sus interacciones lingüísticas. 
Jaramillo Ortegón, Diana, Giraldo Patiño, Milena y Cardona Rivas, Dora (2014). Construcción de la feminidad en adolescentes de La Dorada, Caldas. Ánfora, 21(36), 165-185. Universidad Autónoma de Manizales. ISSN 0121-6538.

\section{Unidad de análisis y unidad de trabajo}

La unidad de análisis fue la construcción de la feminidad de un grupo de 50 adolescentes mujeres entre 12 y 17 años del municipio La Dorada, Caldas (Colombia), de los estratos 1 y 2, del Colegio Renán Barco de los grados $7^{\circ}$ a $11^{\circ}$ de secundaria, y con la "Fundación Apoyar" con sede en el mismo barrio. Las adolescentes presentaban similitudes en la clase social, ciclo de vida, actividad sexual, edad, estado civil y características culturales.

Para la recolección de la información, se adaptaron las guías de entrevistas y grupos focales del estudio La salud sexual y reproductiva de los adolescentes varones y hombres jóvenes de América Latina, realizado por Rebecka Lundgren (2000) para la Organización Panamericana de la Salud -OPS-.

Las entrevistas y los grupos focales fueron grabados y las grabaciones fueron transcritas al pie de la letra por los entrevistadores y revisadas por los supervisores del estudio para asegurar su exactitud. El análisis se realizó por medio de codificación abierta, codificación axial y codificación selectiva (Flick, 2014).

La codificación abierta se realizó sobre las unidades textuales, es decir, sobre los enunciados expresados por los adolescentes con sentido completo. Su propósito fue identificar y denominar los fenómenos apreciables en las unidades textuales (Strauss y Corbin, 2002).

Para ello, se diseñó una matriz de codificación inicial con el propósito de elaborar con eficiencia la codificación abierta, utilizando en algunas ocasiones códigos vivos, es decir, palabras o frases de los entrevistados. Este proceso permite refinar las categorías y orientar el muestreo teórico y la elaboración de una entrevista a profundidad con el fin de realizar la categorización axial. Esto es, formular un grupo de categorías organizadas por importancia, de acuerdo con el objetivo general del estudio, que permitieran interpretar los hallazgos y crear las bases para la formulación de la teoría. Dicha categorización se realizó con base en las precategorías: significado de la feminidad, conocimientos, experiencias e influencias vividas por las adolescentes en el proceso de construcción de su feminidad.

Se avanzó, entonces, al trabajo descriptivo que reunió y organizó la información obtenida, estructurada en un texto que da cuenta de los hallazgos centrales del estudio y confrontado con las adolescentes participantes en el estudio.

El trabajo interpretativo se realizó utilizando la técnica de triangulación de cotejo que consiste en articular:

> Los enunciados de los adolescentes obtenidos a través del grupo focal y la entrevista.

> El conocimiento de las categorías de análisis proveniente de revisiones bibliográficas realizadas.

> La perspectiva de las investigadoras. 


\section{Resultados}

La presentación de los resultados del estudio incluye una descripcióninterpretación, con base en las categorías de análisis: conocimientos y experiencias relacionadas con la feminidad.

Significado de feminidad en las adolescentes

Para las adolescentes de este grupo, las características atribuibles a la feminidad están dadas por los rasgos y las capacidades físicas de procreación, determinadas por su sexo y por las características de personalidad y comportamiento, como lo expresó una participante:

"La diferencia entre un hombre y una mujer es su delicadeza, elegancia y ternura, a diferencia del hombre que se caracteriza por su aspecto brusco y fuerte" (p-7-1.1.2)

Estas características corresponden a opiniones y comportamientos legitimados por factores contextuales y culturales. Se identifica aquí una mezcla entre lo biológico y lo cultural.

Las adolescentes resaltan la importancia de ser madre, del privilegio de dar vida como una de las principales características que las diferencian de los hombres:

"Ser mujer abarca demasiados conceptos como paciencia, ternura, amor incondicional, dar sin esperar, porque tiene el privilegio de dar vida; ser mujer es tener, es soportar y luchar cada día por un espacio y por la superación de la familia, es tener diversos roles como ser ama de casa, madre, esposa, profesional sin derecho a cansancio, sin dejar de ser inteligentes y valientes" (p-11-1.1.2).

Otra participante considera que:

"Ser mujer es tener la capacidad de dar vida, tener sueños y metas para salir adelante con los hijos y con las cosas que uno se proponga" (p-10-1.1.3).

Al resaltar la capacidad biológica de dar vida, las dificultades biológicas para demostrar socialmente dicha capacidad de ser mujer corresponden a los obstáculos que ellas encuentran para demostrar su feminidad:

"Una de mis preocupaciones es no poder ser mama, porque tener hijos es lo que nos hace mujeres ya que los hombres no pueden dar vida" (p-9-2.5.2.).

El vínculo entre ser mujer y ser madre es el eje central de la construcción social del mundo femenino. Según diversos autores con visión tradicionalista de la mujer, ésta nace con la misión de ser madre. Y como madre debe responsabilizarse de sus hijos. Esta postura se deriva de la cultura patriarcal, en la cual la mujer es la cuidadora los hijos y del hogar; es la encargada, por tanto, de las tareas domésticas y su vida se vincula a la del varón. Esto significa que el mundo femenino es un mundo 
privado, doméstico y familiar, es decir, a la esfera reproductiva de la vida. El mundo masculino, en cambio, es abierto al exterior, vinculado a la actividad profesional, al mundo público y a la esfera productiva de la sociedad. En la dimensión personal, la mujer complementa al varón con su amor, sus hijos, sus hijas y su hogar. Así lo expresó una participante:

"El rol de una mujer es ser ama de casa y cuidar del bebe, en cambio el hombre es el que tiene la obligación de la casa" (p-7-1.9.5).

Algunas adolescentes encuentran su esencia de mujer en las cualidades que las diferencian de los hombres:

"Para mí, es ser una persona delicada, ordenada y vanidosa, diferente del hombre, ya que tenemos personalidades distintas que hacen que ellos sean mucho más despreocupados y desordenados con sus cosas".

Otra participante manifestó:

"Ser mujer, significa tener la delicadeza de la mujer, calidad para hacer las cosas, y comportarse de una forma femenina, bonita y atractiva" (p-7-1.1.3).

Una tercera adolescente afirma que ser mujer:

"Es ser delicada, valorándose y queriéndose uno mismo" (p-7-1.1.2).

Esta jovencita alude a la construcción de un autoconcepto positivo.

\section{Experiencias en el proceso de llegar a ser mujer}

Si bien, en un primer momento, las adolescentes se basan en características relacionadas con el comportamiento, la maternidad y el cuidado del hogar, el significado de ser mujer, según las experiencias que contribuyen a dicha feminidad, se evidencia como el desarrollo del cuerpo, las diferencias físicas entre el cuerpo del hombre y de la mujer y la satisfacción con lo físico. Estos son factores importantes para sentirse mujer. A este respecto, se evidencian expresiones como:

"Una mujer y un hombre se diferencian por su sexo, rasgos físicos, comportamientos y lo más importante porque una mujer tiene la capacidad de dar a luz una vida, el hombre no" (p. 7-1.7.4).

O bien:

"Se diferencian en que la mujer tiene vagina y el hombre pene, la mujer tiene senos y el hombre tetillas, la mujer tiene pelo largo y el hombre pelo corto" (p-7-1.7.3). 
Para las adolescentes, las principales experiencias asociadas a la feminidad se expresan, según una participante:

"En los cambios que va teniendo el cuerpo y el cuidado que tengo de él para llegar a ser mujer" (p-7-2.3.2).

Otra se refiere a aspectos como las formas de vestir para resaltar los cambios físicos que ocurrían en su cuerpo:

"Cuando empecé a vestirme como mujer, mostrando los cambios de mi cuerpo y lo bonita que me estaba poniendo" (p-8-2.3.2).

Y otra se refiere a su evolución biológica en relación con la menstruación.

"Cuando me llegó el período y los cambios que tuvo mi cuerpo mostrando que me estaba convirtiendo en mujer” ( $\mathrm{p}-8-2.3 .3$ ).

Para Butler (1998), en el cuerpo se inscriben los valores de género para regular y controlar el comportamiento, las funciones y los espacios que corresponden al cuerpo sexuado, como lo expresó una participante:

"Cuando comencé a atraer a otras personas y a sentirme atraída por otros" (p-8-2.3.5).

Otra lo corrobora así:

"Cuando empecé a sentirme mujer... mostrando los cambios de mi cuerpo porque ya me podía vestir diferente, más sexy y bonita” (p-10-2.3.4).

Y es que el paso de ser niña a ser mujer tiene que ver con los cambios corporales:

"Porque son cambios que a uno lo hacen más mujer y ya comienza a ser bonita para los hombres y comienza a tener novio" (p-9-2.3.3).

Esa disposición corporal a darse, es la que lleva a vincular el inicio de las relaciones sexuales como una experiencia de feminidad:

"Las más significativas, experiencias, son tener relaciones por primera vez, como cuando tuve la primera relación con mi novio (p-8-2.3.1).

El valor del cuerpo como expresión de la feminidad se expresa en su inclusión como preocupación y obstáculo para la feminidad. En este sentido, hay expresiones como la siguiente:

"Me preocupa mi físico, pues soy una niña bastante gordita y aunque hago dieta no bajo de peso, y sé que a los niños les gustan las niñas delgaditas. Por eso, estoy triste porque se burlan de mi cuerpo y porque como mucho" (p-9-2.4.5). 
Esta preocupación es expresada también por otra participante:

"Un obstáculo para mí es mi físico porque soy gordita, y eso preocupa porque las mujeres delgadas son más bonitas, agradables y buscadas” (p-10-2.5.1).

Las imágenes del cuerpo son también objeto de consumo, se incorporan a la experiencia de feminidad los cultos a la mujer delgada y exitosa que impone exclusiones con base en el peso corporal. En esta forma, la insatisfacción con el cuerpo tiene que ver con la percepción de que si no se tienen características físicas "bonitas", las adolescentes, pueden ser castigadas con la escasa atención que se les presta:

"Entre las preocupaciones que existen para llegar a ser mujer estuvo el físico, pero ahora me preocupo mucho más por verme agradable y bonita, con el cabello bonito y bien arreglado y que me vea bien para los demás" (p-10-2.4.3).

De esta manera, hay preocupaciones respecto a la apariencia física:

"Que me engorde, que no me vean bonita y que no me crezcan los senos" (p-7-2.4.3).

Es reiterativa la preocupación por el cuerpo como obstáculo para el desarrollo de la feminidad:

"Que no me siento conforme con mi cuerpo, porque mis senos no crecieron" (p-7-2.5.3).

"Que mi cuerpo no se desarrolle como el de una mujer, que me vea gorda y poco atractiva, porque debo usar ropa grande como de hombre" (p-7-2.5.5).

$\mathrm{El}$ interés por atraer a los hombres fue reiterado entre las adolescentes:

"Mi apariencia y mi estatura, que puede que no le guste a los hombres de mi edad y que me gustan mucho los hombres" (p-8_2.4.1).

"Que mi físico no le guste a los hombres así como mi forma de ser" (p-8-2.4.3).

Estos testimonios indican una clara dependencia de las adolescentes hacia el hombre:

"Me preocupa no hacer feliz a mi novio, que no me vea bonita y suficiente, que no le sea suficiente como mujer" (p-10-2.4.4).

"Acostarse solo con el novio" (p-7-2.3.3).

"Tener novio y vestirme como una señorita para no verme como una niña” (p-7-2.3.5).

Esta preocupación por lo físico se relaciona con los modelos de feminidad que circulan en los medios de comunicación y los pares más cercanos. Por eso, a las adolescentes del presente estudio les preocupa: 
"Que a los hombres no les guste mi cuerpo porque estoy un poco gorda y en la televisión y mis amigas se ven mujeres muy bonitas” (p-8-2.5.1).

A la preocupación por lo físico se suman algunos rasgos relacionados con el comportamiento que se espera de una mujer:

"Mi físico, y que los hombres sientan que a veces soy muy masculina porque soy grosera con los demás. Por eso, tengo que tratar todo el tiempo de tener la cara bonita y hacer sentir bien a mi pareja" (p-8-2.4.5).

La feminidad, en la experiencia, se expresa en cuerpos que modelan su relación con los hombres. Es el cuerpo como centro de una relación íntima con la formación del sujeto femenino que genera, además, una cierta confusión cuando se trasgrede esa relación:

“no sé si me gustan los niños, estoy confundida con mi sexo” (p-7-2.5.2).

"Que sintiéndome mujer me gusten otras mujeres" (p-7-2.4.2).

"Siento que me gustan un poco las niñas, pero también los hombres, por eso no me siento tan femenina” (p-8-2.5.4).

Congruente con las experiencias del cuerpo, en el desarrollo de la feminidad, se encuentra el aprendizaje de comportamientos y conocimientos. Para el aprendizaje de los comportamientos, cobran relevancia, según las adolescentes, los modelos cercanos:

"Se aprende a actuar viendo a las mujeres que a uno lo rodean, como a mi mamá que siempre que sale se arregla muy bien, y yo sigo su ejemplo" (p-7-2.7.1).

"Viendo a los demás, por ejemplo, modelos y actrices, la forma como viven y se relacionan" (p-7-2.7.4).

Para ellas, los conocimientos relacionados con la feminidad incluyen las expresiones propias de la heterosexualidad:

“Como tener sexo y llamar la atención de los hombres" (p-7-2.9.2).

En ellas, se entrecruzan los discursos de la escuela, los programas de salud, las recomendaciones familiares:

"Aprender a llevar una sexualidad responsable y no parecer una mujer que está con todos al mismo tiempo y por eso no la valoran como mujer” (p-7-2.9.3).

“Toca saber qué es lo mejor para las mujeres, para cuidarnos de las infecciones y los embarazos" (p-7-2.9.5). 
La feminidad se relaciona con el paso de niña a mujer porque:

"Ahora creemos que tener relaciones a temprana edad nos hace mujeres más rápido y que los demás nos vean como mujeres" (p-11-2.9.1).

Los caracteres anatomo-fisiológicos que se manifiestan en lo físico y en lo afectivo-emocional ponen en marcha una actividad simbólica frente al devenir de cambios en el sexo femenino y con ello la asunción y la construcción identitaria de una perspectiva de feminidad que se representa y se simboliza, según la experiencia corporal, en modelos que funcionan en forma tácita y silenciosa en la vida cotidiana (Gómez, 1993).

Se trata de una percepción vivencial del propio cuerpo que se halla mediatizada por su efecto social, o la imagen especular que le devuelve el entorno. A muchas personas les es imposible apreciarse a sí mismas en su cuerpo de forma independiente del efecto social, de la presión del grupo social al que pertenecían. En las adolescentes del estudio, la percepción de su cuerpo se halla influida por los sistemas culturales masculinos de referencia.

Por otra parte, junto a las características físicas que determinan el cuerpo de la mujer, ésta debe tener, de acuerdo con el contexto cultural en el que se encuentre, comportamientos que la identifiquen como tal, por lo que debe ser:

"Educada, que no sea grosera y, ante todo, que sea una dama ante los hombres y ante las mujeres, debe ser fiel, amorosa y comprensiva" (p-7-1.2.5).

"Debe actuar con responsabilidad, respeto, amor, cariño, honradez, siendo delicada y tierna, responsable y cumplida” (p-7-1.2.4).

"Debe ser agradable, cariñosa, amable, respetuosa, alegre, juiciosa y buena compañera” (p-7-1.3.1).

Las mujeres se diferencian de los hombres por su comportamiento, y este comportamiento tiene que ver con el conocimiento:

"Los conocimientos que debo aprender es sobre como maquillarme y verme bonita para no parecerme a un hombre y actuar de forma muy femenina y agradable para ello" (p-8-2.2.5).

\section{Influencias en la construcción de la feminidad}

La construcción de la feminidad es un proceso que se inicia en la niñez y transcurre durante toda la vida:

"En todo momento, en todo lugar y en todas las situaciones de la vida, aprendemos a ser mujer" (p-11-3.5.4). 
"Desde niñas debemos aprender a hacer las cosas propias de la mujeres como jugar con muñecas, vestirse bonito, hacerse peinados bonitos y comportarse de una buena forma, que hable de lo femenino que somos y como la mamá nos enseñó a ser" $(\mathrm{p}-8-2.1 .3)$.

"Debemos prepararnos desde pequeñas, porque a ser mujer se aprende durante toda la vida con la forma en como vamos siendo y comportándonos con los demás" $(\mathrm{p}-8-2.1 .5)$.

En el juego con las muñecas hacerse mujer requiere ir...

"asimilando los cambios que de acuerdo a la edad se vayan dando en su cuerpo como lo es su primera menstruación” (p-11-2.3.4).

En el recorrido de niña a mujer, las adolescentes encuentran muchas influencias de la familia, el colegio, los amigos, los medios de comunicación y la cultura:

"La familia es la base donde se aprenden muchos valores, es la primera escuela y de estas bases dependen muchas decisiones" (p-11-3.1.5).

"Es fundamental para nosotras, especialmente el de la mamá para convertirse en mujer" (p-11-3.1.5).

"Las experiencias que marcan son las del colegio. Es allí donde vemos los cambios físicos y emocionales, comienzan a surgir dudas sobre muchas cosas y deseos de experimentar situaciones nuevas que conllevan a diferentes resultados" (p-11-3.1.5).

Los amigos influyen tanto negativa como positivamente. De forma negativa influyen:

"Cuando empiezan a burlarse porque todavía no se han tenidos relaciones sexuales” (p-11-3.2.3).

O pueden ser de gran ayuda cuando:

"Son un soporte con los que comparto y hablo temas que no puedo con mis papás" $(\mathrm{p}-11-3.2 .5)$.

Tradicionalmente, la visión del papel de la mujer ha sido socialmente heredada. Muchas de estas adolescentes actúan basándose en estereotipos familiares y contextuales de acuerdo con las costumbres y los roles de la mujer en su entorno inmediato. Sin embargo, encuentran en los medios masivos de comunicación gran influencia de la forma como les gustaría ser y como a ellas les gustaría ser vistas por la sociedad, lo que ha influido en la transición de la imagen y actuación de la mujer en la sociedad: 
"En este momento los medios de comunicación causan un impacto muy grande en nuestra vida y arrastran mucho, incluso a veces de manera negativa porque tienen mensajes subliminales y nos llevan a veces sin querer a hacer cosas por estar a la moda" (p-11-3.4.3).

Otra participante encuentra esta influencia en los medios de comunicación que:

"Influyen porque nos enseñan el cuidado del cuerpo, el maquillaje y la moda" (p$11-3.4 .5)$.

La construcción de la feminidad está atravesada por influencias múltiples. Estas se evidencian parcialmente en lo enunciado por dos adolescentes:

"Cada día uno aprende a ser mujer y se aprende algo nuevo y nuestro entorno nos enseña cosas nuevas” (p-11-3.5.2).

"En la casa pero es por fuera en donde dejamos ver la educación de los padres y las cosas aprendidas con los amigos y en el colegio" (p-11-3.5.3).

La influencia cultural es reconocida como fuente de aprendizajes:

"En todas las culturas se tienen significados diferentes de respeto por la mujer y así mismo se actúa”, p-11-3.3.2).

"Las mujeres nos desenvolvernos de acuerdo a nuestra cultura y tradiciones" (p$11-3.3 .5)$.

"Creo que influye si lo vemos desde el punto de vista que somos criadas de acuerdo a las tradiciones de nuestras familias, por eso todas somos diferentes" (p-10-3.3.4).

"La Cultura influye mucho en las mujeres ya que todas nos comportamos de forma diferente de acuerdo a las tradiciones y a las características de los pueblos y de las ciudades, por ejemplo en Bogotá las mujeres son más aburridas y recatadas” (p-83.3.1).

Se reconoce, entonces, que cada cultura teje las creencias relacionadas con el papel que las mujeres juegan en la sociedad o que da lugar a los estereotipos sobre el género. Para Lagarde “ los estereotipos sociales y sus normas, son fundamentales en la configuración de la subjetividad de cada quien y en la cultura” (1996:13-38)

\section{Conocimientos en la construcción del significado}

Para las adolescentes que participaron en este estudio, la construcción de la feminidad se relaciona con su percepción del cuerpo, de la sexualidad y de los conocimientos que se van adquiriendo. Respecto a los conocimientos, el proceso se inicia muy temprano: 
"Los conocimientos que me han enseñado en la escuela, el colegio, mi mamá, mis tías” (p-8-2.2.2).

Con ello, esta participante hace referencia a los conocimientos sobre el cuerpo, la manera de vestirse, maquillarse y comportarse como una mujer.

En la valoración de los saberes relacionados con el proceso de hacerse mujeres, las adolescentes destacan los pertinentes a la sexualidad:

"porque así nos identificamos y nos damos cuenta que si nos gustan los hombres $\mathrm{y}$ uno siente cosas por dentro que nos hacen desearlo y querer estar con ellos" (p-82.9.1).

"A veces hay que demostrar que ya somos grandes y que somos mujeres consiguiendo novio y teniendo relaciones con él y haciendo las cosas que las mujeres hacen, es un proceso" (p-8-2.9.2).

"porque aunque queramos estar con muchos hombres, el conocer nuestra sexualidad nos da muchos conocimientos sobre cómo debemos comportarnos para ser mujeres" (p.8-2.9.3).

Tales conocimientos hacen parte de los discursos sobre la planificación, el cuidado del cuerpo y la prevención de enfermedades de trasmisión sexual:

"puede estar relacionado el cómo llegar a ser mujer con los conocimientos sobre sexualidad, ya que por medio del conocimiento sobre sexualidad podemos planificar y decidir cuándo queremos ser mamás y dejarnos de ver como jóvenes para vernos como mujeres" (p-11-2.9.5).

"Pienso que sí, ya que cuando pasamos de niña a mujer debemos protegernos y cuidarnos, e ir conociendo nuestro cuerpo y sus cambios para evitar por ejemplo enfermedades de trasmisión sexual o un embarazo no deseado, o las cosas que nos gustan y las que no" (p-10-2.9.2).

"Porque si aprendemos a conocer nuestro cuerpo y saber cómo cuidarnos y protegernos aprendemos a ser mujer” (8_2.9.4).

Las experiencias, los conocimientos y las influencias que viven las adolescentes con las que se realizó el presente estudio permiten sustentar que el proceso de construcción de la feminidad en un grupo de adolescentes del municipio de La Dorada es una experiencia corporal subordinada a las representaciones masculinas. Esa experiencia se describe en el siguiente aparte. 
Jaramillo Ortegón, Diana, Giraldo Patiño, Milena y Cardona Rivas, Dora (2014). Construcción de la feminidad en adolescentes de La Dorada, Caldas. Ánfora, 21(36), 165-185. Universidad Autónoma de Manizales. ISSN 0121-6538.

\section{Construcción de la feminidad en adolescentes: una experiencia corporal subordinada a la masculinidad}

En los resultados obtenidos, se observa que la construcción de la feminidad en este grupo de adolescentes, es un proceso a través del cual se apropian de una imagen de la sexualidad elaborada por los hombres sobre las mujeres. En ese proceso, se identifican dos tipos de mediaciones: una mediación cultural y una mediación personal, como lo propone Colás (2007).

Respecto de la mediación cultural, la construcción de la feminidad en un grupo de adolescentes de La Dorada es un proceso que reconoce diferentes niveles de interacción, mediada por conocimientos, significados y experiencias que hacen parte del entorno familiar y escolar en un primer momento, y de los instrumentos simbólicos proporcionados por la cultura, en un segundo momento. Para Brofenbrenner (1973), los modos de ser, de vestir, de lucir, de parecer articulan de manera concéntrica y autocontenida el microsistema, el mesosistema y el macrosistema o ambiente ecológico en el que se configura el desarrollo de las personas.

En el proceso de construcción de la feminidad, el microsistema está representado por las actividades, los roles, las formas de vestir y las relaciones propias de la mujer que, principalmente, la madre y otros miembros de la familia van imprimiendo en el entorno en que la niña nace y en el que trascurren sus primeros años. Desde que nacen, las niñas se exponen a modelos y reacciones que las convencen de que a las diferencias biológicas y sexuales les corresponden las diferencias psicológicas y estéticas y así aprenden a comportarse de acuerdo con las normas del contexto cultural en el cual crecen.

Ha sido ampliamente documentado que, aún a edades muy tempranas, las niñas y los niños ya van estableciendo los estereotipos de género, similares a los de las personas adultas (Espinoza, 2005). Según Lamas, citado por Esther Pineda (2011), el hecho de que mujeres y hombres sean diferentes, anatómicamente, los induce a creer que sus valores, cualidades intelectuales, aptitudes y actitudes también lo son.

Es en estos espacios en que los sentimientos maternales se expresan como necesidades orgánicas, que niegan el carácter opcional de la maternidad, naturalizando y universalizando esta institución social que suscita para el futuro, en las adolescentes, una diferenciación de la mujer con propósitos creadores, de una construcción natural de feminidad para ser mamá: ser mamá es parte de la naturaleza femenina en el común de las mujeres.

El hecho de que se siga planteando la cuestión del género según las diferencias sexuales, se mantiene una oposición conceptual en los discursos culturales dominantes y sus expresiones biológicas, médicas, legales, filosóficas o literarias que alimentan la tendencia a reproducirse o a retextualizarse, como señala De Lauretis (1989).. 
La adhesión a esta diferenciación, para Bourdieu (2000), responde a una sumisión inmediata y prerreflexiva de los cuerpos socializados y no a una decisión deliberada e ilustrada. Esto, porque sus pensamientos y sus percepciones "están estructurados de acuerdo con las dominación que se les ha impuesto, sus actos de conocimiento son, inevitablemente, unos actos de reconocimiento, de sumisión” (Bordieu, 2000, p. 26).

En el mesosistema, se entrecruzan las múltiples relaciones en las que una niña participa en la escuela, las relaciones interpersonales con las amigas y los amigos, cuando puede hablar con ellos y discurrir sobre aspectos de su sexualidad que no puede hacer con su madre o sus familiares. Se observa que el escenario de la escuela y las interacciones que allí se dan, desempeñan un papel relevante en este proceso.

Se ha reconocido ampliamente que las personas interactúan y aprenden de sus pares. Al respecto, Bearman y Brückner (1999) encuentran que un alto nivel de interacciones sociales en los colegios tiene efectos significativos en el inicio de relaciones a edades tempranas.

Los pares adolescentes, hombres y mujeres, promueven interacciones sociales en las que las mujeres se inclinan a tratarse a sí mismas como objetos estéticos que suscitan admiración y deseo. En palabras de Bourdieu:

Por eso, y más que en sociedades arcaicas, las mujeres están colocadas en el ámbito del parecer, del ser percibido, de complacer y les incumbe volverse seductoras mediante un trabajo cosmético que, en ciertos casos, y sobre todo en la pequeña burguesía de representación, constituye una parte muy importante de su trabajo doméstico..." (Bordieu, 1980, p. 43).

En el macrosistema, la niña se expone al imaginario social y cultural de género que se traduce en patrones de conducta, roles, ritos y funciones diferenciadas según el sexo, que se expresan en esta investigación en un tipo de mujer que debe tener rasgos de una belleza necesaria para ser visibilizada y ser atractiva para el hombre. Se trata de una feminidad y una masculinidad dicotomizadas por el modelo hegemónico que circula en las culturas.

En relación con la mediación personal, en diálogo con la mediación cultural, las adolescentes legitiman en su cuerpo la experiencia como un complejo de efectos de significado, hábitos, disposiciones, asociaciones y percepciones resultantes de la interacción de significaciones del yo y del mundo externo, lo que configura una existencia en la que lo cognitivo, lo afectivo y lo conductual se orientan en función del varón.

Los procesos psicofísicos y la presión social se expresan en la persona y, aquí, el cuerpo es lenguaje que le habla a los otros y a sí misma. Se trata de una experiencia tal que, al no sentirse admirada por el varón se experimenta el temor de la soledad y la imposibilidad de la realización femenina en la maternidad. Este temor ocurre también al sentirse diferente a las demás. El cuerpo es especial en las adolescentes 
y juega un papel crucial y a veces negativo. Por el miedo al rechazo dedican parte de su vida a parecer aceptables, particularmente para los hombres, y asumen una dinámica que tiende a legitimar una apreciación de sí mismas basada en el cuerpo, dependiente del efecto social y la presión del grupo social.

En esta forma, se sostiene que la mujer es para darse a otros, sobre todo a un hombre.

Es evidente que quienes perciben el rechazo social hacia la feminidad o la masculinidad, expresan la angustia que aparece al traspasar las fronteras hacia otro territorio de género que da la medida de las constricciones sociales. Espinosa (2005), señala que la intolerancia ante las transgresiones de los roles propios de la feminidad durante la adolescencia hacen parte de una intensificación del sexo hacia la reconstrucción de la identidad que tuvo lugar en la primera infancia. Esta intensificación se basa en la percepción de que en el cuerpo sienten las adolescentes el paso de ser niña a ser mujer.

\section{Conclusiones}

El estudio permitió dilucidar la manera como operan las relaciones socioculturales en las que deviene la construcción de la feminidad por las adolescentes. Ésta, emerge de dos procesos íntimamente ligados y diferenciables. El primero, se asimila a la construcción histórica que inicialmente basada en el morfismo sexual, avanza hacia la perspectiva de género como una realidad social objetivada esencial e inmutable para el desarrollo funcional de los sexos. El segundo, proviene de su experiencia vital, íntima y personal de experimentar su cuerpo, inicialmente como objeto de atención de sí misma y de los demás, especialmente del varón, como su objeto de deseo. Su entrega es la máxima experiencia de la feminidad y es el objetivo necesario para la reproducción y la maternidad.

En los dos procesos mencionados lo femenino es subordinado al modelo masculino y mantiene la asimetría del acceso a la libertad en todas las esferas del desarrollo humano, al poder socio-cultural, económico y político por la mujer. No obstante, en la complejidad de la constitución de la feminidad, es la dinámica de la evolución del cuerpo como objeto de deseo lo que constituye la esencia de la feminidad.

Por eso a partir del estudio se arriesga la hipótesis de que postular a la mujer como sujeto en la construcción de nuevas subjetividades, a través de una práctica reflexiva sobre los experiencias ancladas en la historia familiar y social, contribuye a la deslegitimación de la subordinación que aún pesa sobre ellas.

Y es que frente a un proceso de dominación masculina que se vive desde la primera infancia, emerge la necesidad de una táctica política de potenciación de lo femenino, en la construcción de una identidad fluida, versátil, sin fronteras, abierta a nuevas posibilidades y con potencial para resignificar el mundo adolescente y adulto. 


\section{Referencias}

Bearman, P., y Brückner, H. (1999). Peer Effects on Adolescent Sexual Debut and Pregnancy: An Analysis of a National Survey of Adolescent Girls. En P. B. (Eds.). Power in Numbers: Peer Effects on Adolescent Girls' Sexual Debut and Pregnancy. Capítulo 1. (pp. 7-26). New York: The National Campaign for the Prevention of Teen Pregnancy.

Bourdieu, P. (1980). La eternización de lo arbitrario. Barcelona: Anagrama.

Bourdieu, P. (2000). La dominación masculina. Barcelona: Anagrama.

Bronfenbrenner, U. (1973). Influencing Human Development .Ecological models of human development. En international Encyclopedia of Education. New York: Editorial Oxford.

Butler, J. (1998). La Temática del Cuerpo Femenino. Barcelona: Paidós.

Colás, P. (2007). La construcción de la identidad de género: enfoques teóricos para fundamentar la investigación e intervención educativa. Universidad de Sevilla $25(1), 151-166$.

De Lauretis, T. (1989). La tecnología del género. En Technologies of Gender: Essays on Theory, Film and Fiction. London: Macmillan.

Del Castillo, S., Roth, A., Wartki, C., Rojas, R., y Chacón, O. (2008). La implementacion de la política pública de salud sexual y reproductiva (SSR) en el Eje Cafetero Colombiano: el caso del embarazo adolecente. Revista latinoamericana de ciencias sociales, niñez y juventud 6(1), 217-255.

Espinosa, M. A. (2005). Roles de Género y Modelos Familiares. Recuperado de http://www.emakunde.euskadi.net/u72-publicac/es/contenidos/ informacion/pub_jornadas/es_emakunde/adjuntos/sare2005_es.pdf

Flick, U. (2004). Introducción a la investigación cualitativa. Madrid: Morata.

Flórez, C., y Soto, V. (2013). Serie de Estudios a Profundidad. Factores protectoes y de riesgo de embarazo adolescente en Colombia. Bogotá : Profamilia: http:// www.profamilia.org.co/encuestas/Profamilia/Profamilia/images/stories/ ENDS\%201990\%20-2010/Estudio\%20.

Glaser, B., y Strauss, A. (1967). El desarrollo de una teoría fundamentada. San Francisco: Escuela de enfermería, Universidad de California.

Gómez, G. (1993). Apuntes para el Trabajo en Sexualidad desde los Enfoques de Género, Derechos y Diversidad. Montevideo: Fondo de Población de las Naciones Unidas. 
Lagarde, M. (1996). El género en: Género y Feminismo. Desarrollo Humano y Democracia. España: Ed. Horas y Horas

Lundgren, R. (2000). Protocolo de investigación para el estudio de la salud sexual y reproductiva de los adolescentes varones y hombres jóvenes en América Latina. San José: Organización Panamericana de la Salud OPS.

Pineda, E. (2011). Roles de Género y Sexismo en Seis Discursos sobre la Familia Nuclear. Caracas: Ediciones Acercándonos, Universidad Central de Venezuela, Departamento de Ciencias Sociales.

República de Colombia (2003). Política Nacional de Salud Sexual y Reproductiva, Bogotá DC.

República de Colombia, Ministerio de la Protección Social (2007). Plan Nacional de Salud pública 2007-2010. Bogotá DC

Steve, J. (2005). Estilos Parentales, Clima Familiar y Autoestima en Adolescentes. Valencia: Universidad de Valencia, España. Departamento de Psicología Social.

Strauss, A., y Corbin, J. (2002). Bases de la Investigación Cualitativa. Técnicas y Procedimientos para Desarrollar la Teoría Fundamentada. Bogotá: Contus . 